\title{
Geomagnetic Effects of the Equatorial Counter-Electrojet and Their Extension to Non-Equatorial Latitudes
}

\author{
N. S. SASTRI and B. N. BHARGAVA \\ Indian Institute of Geomagnetism, Colaba, Bombay, India \\ (Received October 25, 1978; Revised October 1, 1979)
}

\begin{abstract}
Variation of the additional magnetic field due to the counter-electrojet is examined over the latitudes from the dip equator to Sabhawala (dip= $45^{\circ}$ ) separately for January and the summer months. The additional field is derived from a set of days on which intense afternoon counter-electrojet events have been recorded at Trivandrum near the dip equator, during the period 1965-1977. An almost a reversal in the pattern of field variation in declination is observed between January and summer at all the stations. An approximate representation of the overhead current vectors during the daytime hours in summer months, under the assumption that the field effects result from an additional current system, suggests a vortex in the afternoon with a clockwise rotation and an oppositely directed vortex in the forenoon.
\end{abstract}

\section{Introduction}

The abnormal depression in the variation of the horizontal component, $H$, of the geomagnetic field at stations in the equatorial electrojet belt, often observed clearly in the afternoons on relatively quiet days during periods of low solar activity, are believed to be associated with a narrow band of westward current called the equatorial counter-electrojet (GouIN and MAYAUD, 1967). This depression in $H$ is generally confined to the field in the electrojet region. Corresponding variations in the vertical component, $Z$, are pronounced at stations located at the fringe of the electrojet belt where the signature of the equatorial electrojet in $Z$ is known to be the largest. Effects in declination, $D$, are comparatively small. MAYAUD (1977) has recently given a review of the geomagnetic aspects of the equatorial counter-electrojet. $\mathrm{He}$ cites a few examples of the effects in $D$ and $Z$ seen at latitudes farther away from the electrojet belt. Examining magnetic variations during the reverse equatorial electrojet events STENING (1977) finds, in most cases, departures from the normal variation at non-equatorial latitudes in the same longitude zone as the equatorial station. Considering the observed variation in the equatorial field as a resultant of two components: (i) quiet-day $S q$ plus electrojet field and (ii) an additional field presumably due to the counter-electrojet, the nature of the additional field in $H$ in the Indian 
region was examined in an earlier paper (BHARgaVA and SASTRI, 1977). Adopting the same procedure, the additional field in $D$ and $Z$ at stations extending over the latitudes from the equator to $30^{\circ} \mathrm{N}$ in the Indian region is derived here from a set of days of intense afternoon counter-electrojet events.

\section{Method}

After selecting the day on which an intense counter-electrojet (C-E) afternoon event has occurred, a control day, free from the afternoon C-E effect, with index $A p$ nearly the same as for the C-E day is chosen as close as possible to the selected C-E day to ensure similarity in the seasonal, solar cycle and magnetic activity-associated components between the 2 days. Assuming that the field on the control day is associated with the quiet-day $S q$ and electrojet, the difference between the corresponding hourly mean values of the 2 days, derived by subtracting the night-time base value after correcting for the non-cyclic change, may be considered to represent the additional field at each hour of the C-E day.

\section{Analysis}

Fourteen days of intense counter-electrojet events in the afternoon hours were selected from records over the period 1965-1977; of these 9 days were in the month of January and 5 days in the northern summer months, May to August. The average $A p$ index was 5.5 for the C-E day and 3.0 for the control day for the January events and, 3.4 and 3.0 respectively for the events in the summer months. The average variations in all the three elements, $D, H$, and $Z$, on the day of the C-E event and the control day at the six observatories in the Indian region are presented in Fig. 1 for January and in Fig. 2 for the northern summer months. The co-ordinates of the

Table 1. Location of the observatories.

\begin{tabular}{|c|c|c|c|c|c|c|}
\hline \multirow{2}{*}{ Observatory } & \multirow{2}{*}{$\begin{array}{l}\text { Year of } \\
\text { starting }\end{array}$} & \multicolumn{2}{|c|}{ Geographic } & \multicolumn{2}{|c|}{ Geomagnetic } & \multirow{2}{*}{ Dip } \\
\hline & & Latitude & Longitude & Latitude & Longitude & \\
\hline Trivandrum & 1957 & $8^{\circ} 29^{\prime} \mathrm{N}$ & $76^{\circ} 57^{\prime} \mathrm{E}$ & $1.2^{\circ} \mathrm{S}$ & $146.4^{\circ}$ & $-1^{\circ}$ \\
\hline Kodaikanal & 1949 & $10^{\circ} 14^{\prime} \mathrm{N}$ & $77^{\circ} 28^{\prime} \mathrm{E}$ & $0.6^{\circ} \mathrm{N}$ & $147.1^{\circ}$ & $3^{\circ}$ \\
\hline Annamalainagar & 1957 & $11^{\circ} 22^{\prime} \mathrm{N}$ & $79^{\circ} 41^{\prime} \mathrm{E}$ & $1.4^{\circ} \mathrm{N}$ & $149.4^{\circ}$ & $5^{\circ}$ \\
\hline Hyderabad & 1964 & $17^{\circ} 25^{\prime} \mathrm{N}$ & $78^{\circ} 33^{\prime} \mathrm{E}$ & $7.6^{\circ} \mathrm{N}$ & $148.9^{\circ}$ & $20^{\circ}$ \\
\hline Alibag & 1905 & $18^{\circ} 38^{\prime} \mathrm{N}$ & $72^{\circ} 52^{\prime} \mathrm{E}$ & $9.5^{\circ} \mathrm{N}$ & $143.6^{\circ}$ & $24^{\circ}$ \\
\hline Ujjain & 1975 & $23^{\circ} 11^{\prime} \mathrm{N}$ & $75^{\circ} 47^{\prime} \mathrm{E}$ & $13.5^{\circ} \mathrm{N}$ & $147.0^{\circ}$ & $33^{\circ}$ \\
\hline Jaipur & 1975 & $26^{\circ} 55^{\prime} \mathrm{N}$ & $75^{\circ} 48^{\prime} \mathrm{E}$ & $17.3^{\circ} \mathrm{N}$ & $147.4^{\circ}$ & $40^{\circ}$ \\
\hline Sabhawala & 1964 & $30^{\circ} 22^{\prime} \mathrm{N}$ & $77^{\circ} 48^{\prime} \mathrm{E}$ & $20.8^{\circ} \mathrm{N}$ & $149.8^{\circ}$ & $45^{\circ}$ \\
\hline
\end{tabular}


stations are given in Table 1. The average C-E effects, derived from Figs. 1 and 2, are shown in Fig. 3. The time-scale in the graphs refers to $75^{\circ}$ East Meridian Time which approximately represents the local time within few minutes at all the stations. Note that $Z$ curves at Trivandrum are reversed in all the figures.

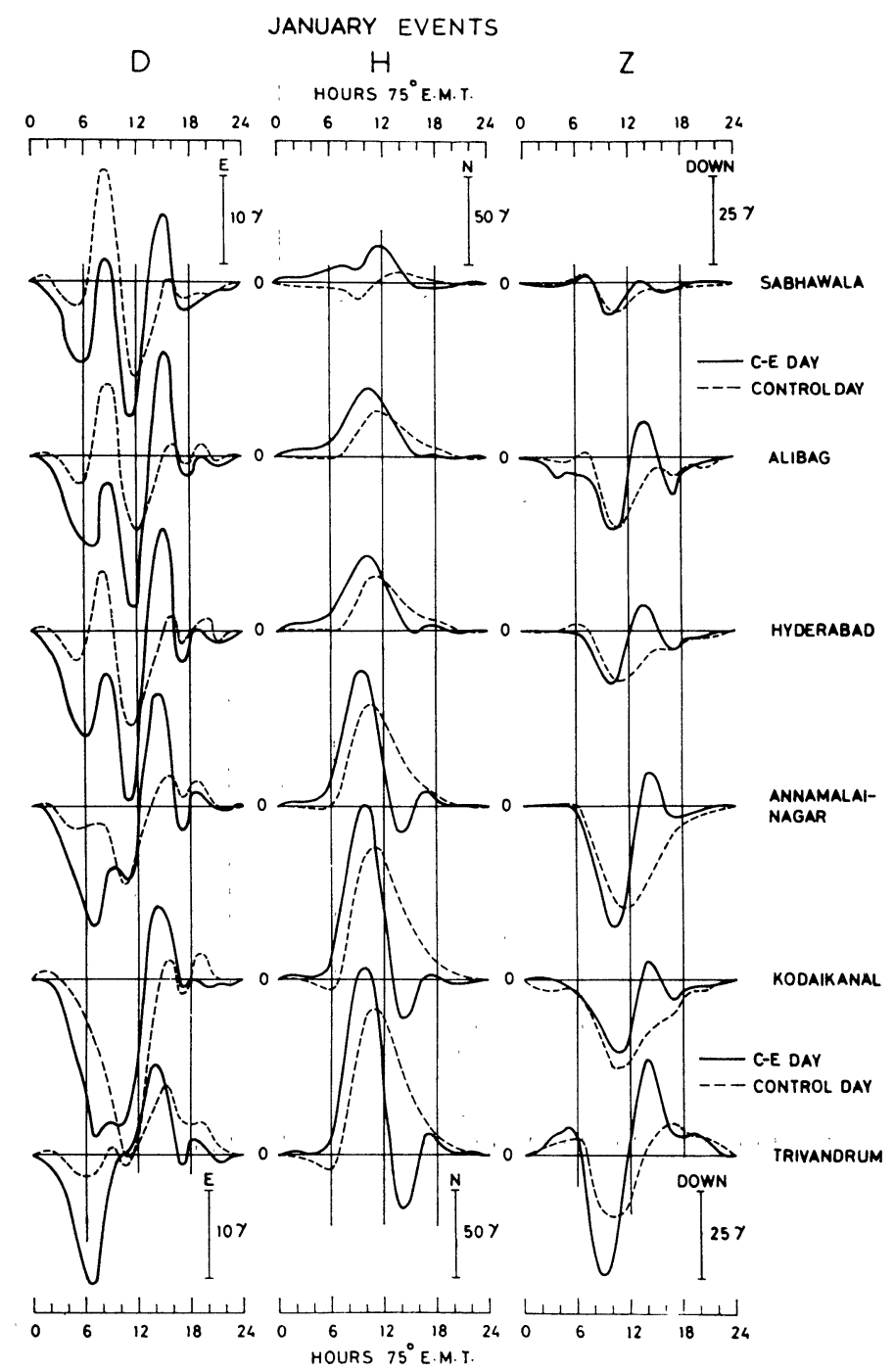

Fig. 1. The average variation in declination, horizontal and vertical intensities, $D, H$, and $Z$, on the day of the afternoon counter-electrojet event (solid line) and on the control day (broken line) for 9 intense events in January over the period 1965-1977. Note that $Z$ curve of Trivandrum is plotted reverse. 


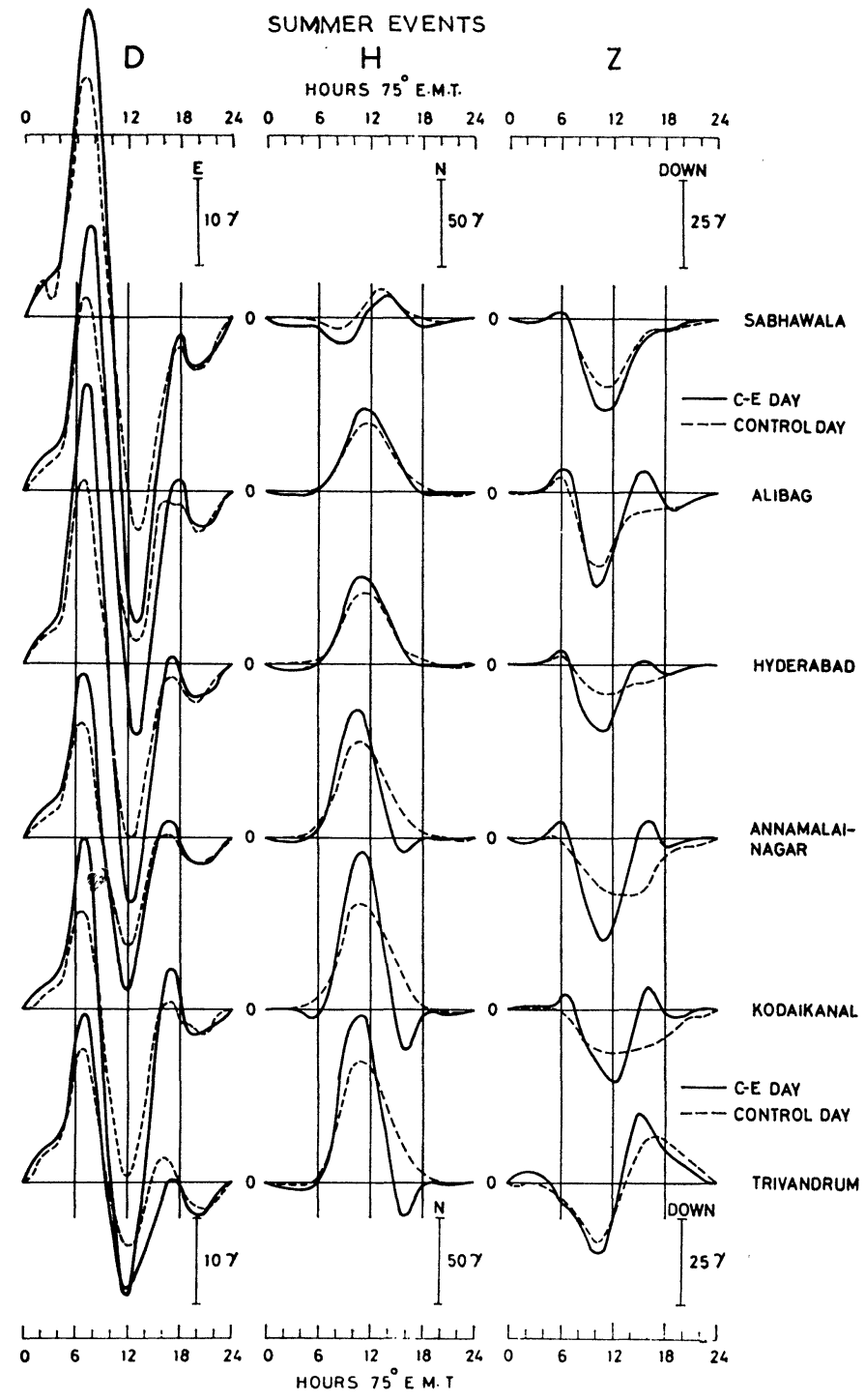

Fig. 2. The average variation in declination, horizontal and vertical intensities, $D, H$, and $Z$, on the day of the afternoon counter-electrojet event (solid line) and on the control day (broken line) for 5 intense events during the northern summer months, May to August, over the period 1965-1977. Note that $Z$ curve of Trivandrum is plotted reverse.

\section{Results}

Some salient features of these variations are as follows. 


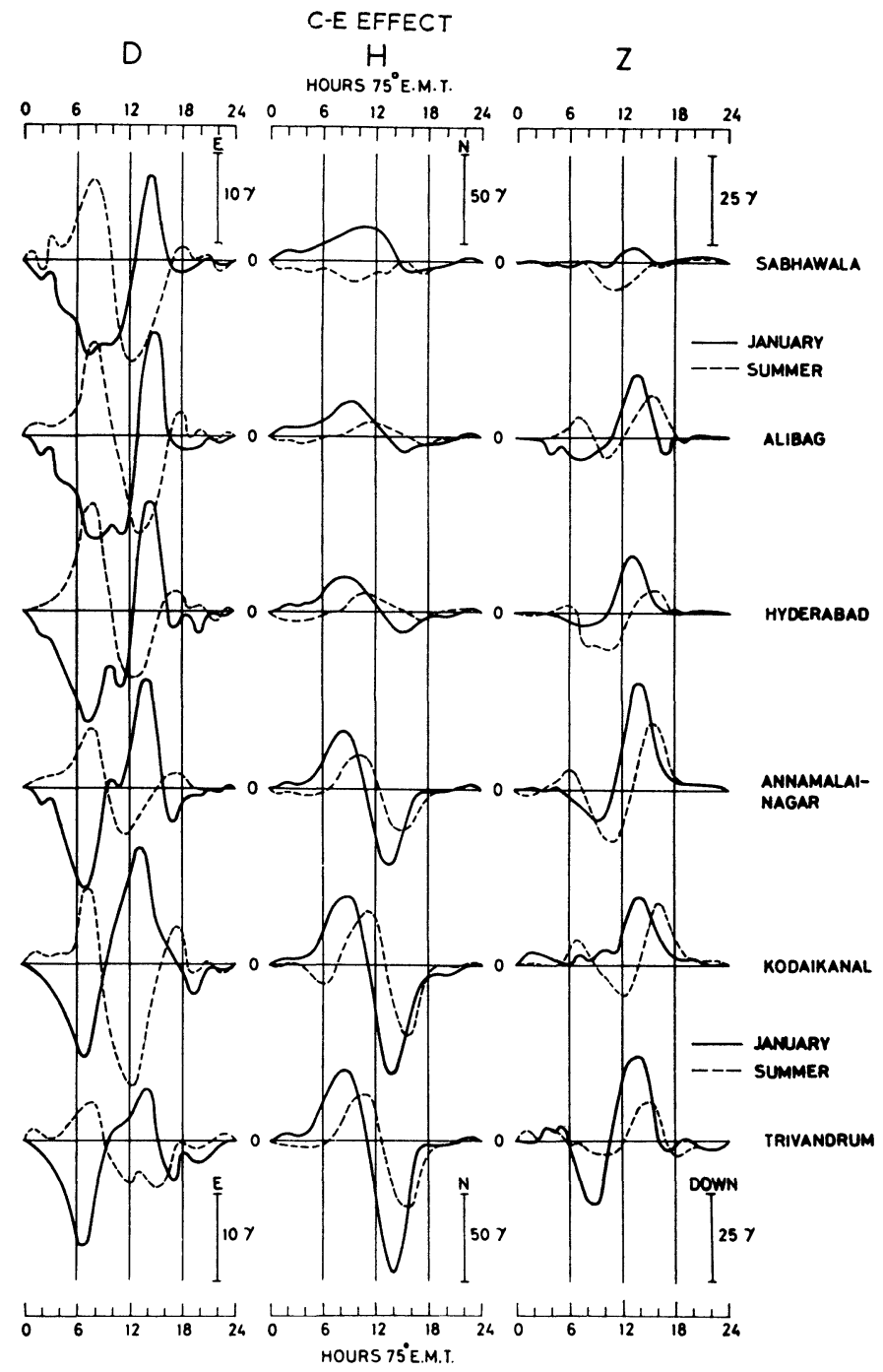

Fig. 3. The average additional field in declination, horizontal and vertical intensities, $D, H$, and $Z$, on the day of afternoon counter-electrojet event during January (solid line) and during summer (broken line) obtained by subtracting the variation on the control day from the variation on the day of counter-electrojet event in Figs. 1 and 2 . Note that $Z$ curve of Trivandrum is plotted reverse.

\subsection{Declination}

For the January events (Fig. 1) the forenoon pattern on the C-E day remains the same as that on the control day but appears to be superposed upon by an addition- 
al westward field with its maximum between 06 and $07 \mathrm{hr}$, seen clearly in Fig. 3. The forenoon peak, on both C-E day and the control day, is observed only outside the electrojet belt. In the afternoon, the increase in easterly declination is rapid on the C-E day, attaining its maximum about an hour earlier than on the control day.

In the summer months (Fig. 2) the pattern of variation on the C-E day remains exactly the same as on the control day.

\subsection{Horizontal intensity}

The forenoon maximum on the C-E day in January occurs about an hour earlier than on the control day and after attaining the maximum, the decrease of the northward field is rapid on the C-E day. For the summer months, the variations on the C-E day and the control day are almost the same except for the rapid decrease of the northward field after attaining the maximum in the forenoon on the C-E day.

\subsection{Vertical intensity}

The variation in the afternoon on the C-E day differs significantly from that on the control day upto the latitude of Alibag both in January and the summer months.

\subsection{C-E effects}

The striking feature of the $D$ graphs in Fig. 3 is the reversal of the pattern of variation between January and the summer months at all the stations from Trivandrum to Sabhawala; variation in January resembles the quiet-day variation, $S q$, in the southern hemisphere in that month. The departure of the variation in declination on the C-E day from that on the control day is statistically significant between 04 and $08 \mathrm{hr}$ and again around $14 \mathrm{hr}$ in January, but only for a short interval in the forenoon around $07 \mathrm{hr}$ in the summer months.

The positive salient in $H$ is significantly different from zero at all the stations in January, but only at the electrojet stations in the summer months. It is to be noted that in summer months Sabhawala shows significant southward field in the forenoon when the field under the electrojet is northward. The afternoon depression is found prominent only in the electrojet belt, with its maximum occurring earlier in winter than in summer as noted by RASTOGI (1973). Large counter-electrojet effects in $Z$ are seen at Annamalainagar, as expected, and the effects extend up to Alibag.

MAYAUD (1977) observes in $D$ and $Z$ small but significant variations in curves similar to those in Fig. 3, obtained by him for stations farther from the equatorial latitudes. These variations provide an indication about the direction of rotation of the current vortices.

The distribution of the monthly frequencies of C-E occurrence indicates (BHARGAVA and SASTRI, 1979) a broad maximum in the June solstitial months with a secondary but well-defined and narrow maximum in January; the minima in March and November-December are relatively sharp. As seen from Figs. 1, 2, and 3, a 


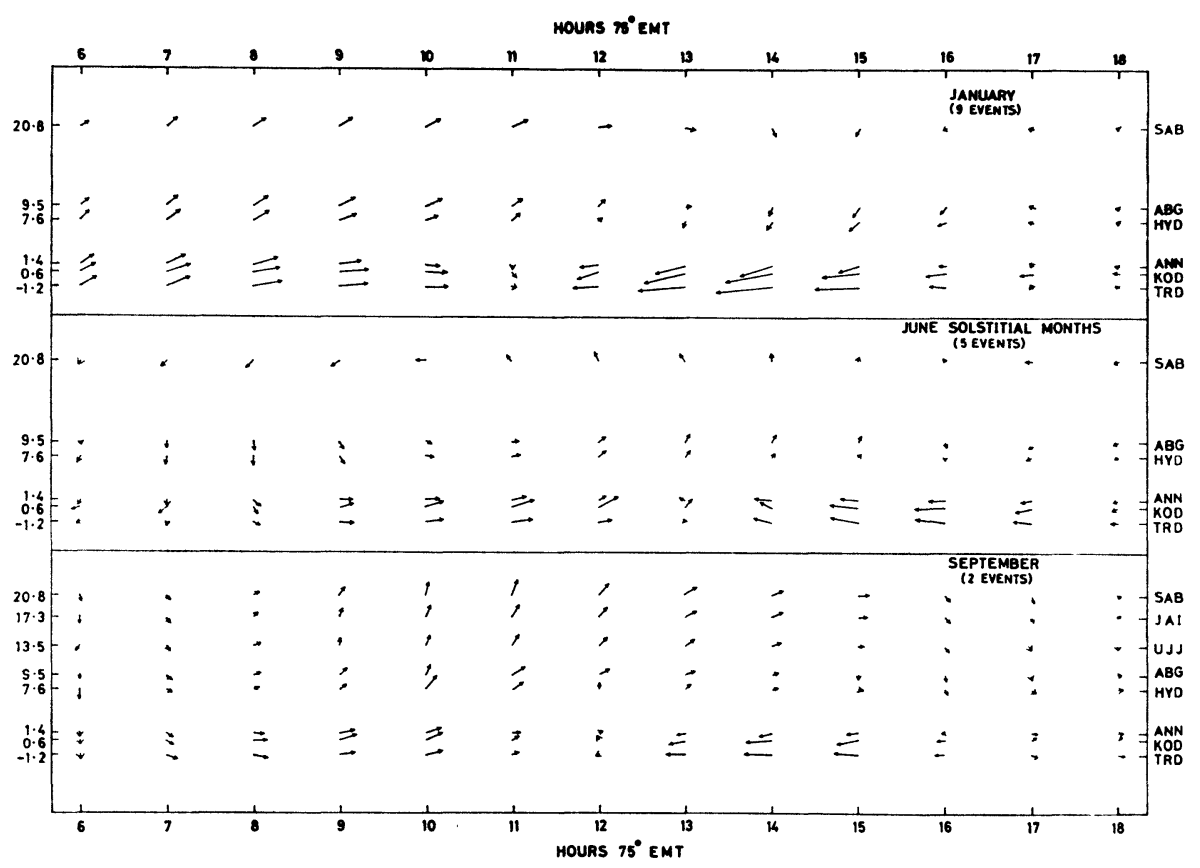

Fig. 4. An approximate representation of the equivalent current vectors of the average counter-electrojet currents in the Indian region in January (9 events), summer ( 5 events), and in September ( 2 events). Geomagnetic latitude of the station is given on the left side.

characteristic difference appears to be present between January and the summer months in the variations associated with the counter-electrojet, especially in declination. A better picture of this difference between January and the June solstitial months is given in Fig. 4, which is an approximate representation of the horizontal current vectors during day-time hours ( 06 to $18 \mathrm{hr}$ ) over each of the stations obtained by combining the corresponding variations in $H$ and $D$ due to the counter-electrojet, shown in Fig. 3. Similar diagram for an average of two events in September is also shown in the figure. If the C-E effects are assumed to result from an additional current system in the dynamo region of the ionosphere, separate from the $S q$ current system, then this additional current system appears to have a vortex in the afternoon side with currents flowing in the clockwise direction and its centre probably located between Alibag and Trivandrum. Another vortex with the currents flowing in an anticlockwise direction appears in the forenoon during the June solstitial months with its centre located between Alibag and Sabhawala; the centre seems to shift to an earlier hour and to higher latitudes in the month of September. The situation in January looks different indicating a single vortex a little after noon with currents 
flowing in the clockwise direction and its centre located probably at about the latitude of Alibag. However, the eastward current along the geomagnetic equator in January is strong in comparison with its intensity in the June solstitial months and September. In addition, there is an intense westward declination change on C-E days in January with a maximum around $8 \mathrm{hr}$ LT (Fig. 3) at all the stations. These features suggest that, from evidence derived from Indian data, the existence of a large scale counter-clockwise current vortex in the forenoon hours in January also cannot be ruled out. This could be verified from an examination of simultaneous records in middle latitudes north of India. A definitive statement on the absence or presence of counter-clockwise current vortex in January is possible only after we know whether or not a pair of clockwise and counter-clockwise current vortices appear in the "forenoon" counter-electrojet events also. It is proposed to carry out such an analysis in the near future.

STENING (1977) has calculated current patterns using various wind systems in the dynamo region that might produce the observed variations on the days of afternoon counter-electrojet events. But he finds that different wind systems are required on different occasions. It is possible that the causative factor for the C-E events may be different at least in January from that in the other months. MAYAud (1977) has indeed suggested tentatively two sources of C-E events: (i) a source centred on the month of January at any longitude and strongly modulated by the moon, and (ii) a source centred on the summer solstice in each hemisphere. On some occasions the C-E effect observed in one longitude zone is not seen on the same day in another longitude zone even only $30^{\circ}$ away (RASTOGI, 1973) which implies that on some occasions the patterns of current vectors indicated in Fig. 4 may not persist even for $2 \mathrm{hr}$.

We thank Professor Satya Prakash, Physical Research Laboratory, Ahmedabad, for helpful discussions.

\section{REFERENCES}

Bhargava, B. N. and N. S. SAStri, A comparison of days with and without occurrence of counterelectrojet afternoon events in the Indian region, Ann. Géophys., 33, 329-332, 1977.

Bhargava, B. N. and N. S. SaStri, Some characteristics of the occurrence of the afternoon counterelectrojet events in the Indian region, J. Geomag. Geoelectr., 31, 97-101, 1979.

Gouin, P. and P. N. Mayaud, A propos de l'existence possible d'un 'contre electrojet" aux latitudes magnetiques equatoriales, Ann. Géophys., 23, 41-47, 1967.

Mayaud, P. N., The equatorial counter-electrojet-A review of its geomagnetic aspects, J. Atmos. Terr. Phys., 39, 1055-1070, 1977.

Rastogi, R. G., Counter equatorial electrojet currents in the Indian zone, Planet. Space Sci., 21, 1355-1365, 1973.

Stening, R. J., Magnetic variations at other latitudes during reverse equatorial electrojet, J. Atmos. Terr. Phys., 39, 1071-1077, 1977. 